\title{
Dynamical effects in fusion with exotic nuclei
}

\author{
K. Vo-Phuoc, ${ }^{*}$ C. Simenel, and E. C. Simpson \\ Department of Nuclear Physics, Research School of Physics and Engineering, Australian National University, \\ Canberra, Australian Capital Territory 2601, Australia \\ (Received 23 June 2016; published 15 August 2016)
}

\begin{abstract}
Background: Reactions with stable beams have demonstrated strong interplay between nuclear structure and fusion. Exotic beam facilities open new perspectives to understand the impact of neutron skin, large isospin, and weak binding energies on fusion. Microscopic theories of fusion are required to guide future experiments.

Purpose: To investigate new effects of exotic structures and dynamics in near-barrier fusion with exotic nuclei. Method: Microscopic approaches based on the Hartree-Fock (HF) mean-field theory are used for studying fusion barriers in ${ }^{40-54} \mathrm{Ca}+{ }^{116} \mathrm{Sn}$ reactions for even isotopes. Bare potential barriers are obtained assuming frozen $\mathrm{HF}$ ground-state densities. Dynamical effects on the barrier are accounted for in time-dependent Hartree-Fock (TDHF) calculations of the collisions. Vibrational couplings are studied in the coupled-channel framework and near-barrier nucleon transfer is investigated with TDHF calculations.

Results: The development of a neutron skin in exotic calcium isotopes strongly lowers the bare potential barrier. However, this static effect is not apparent when dynamical effects are included. On the contrary, a fusion hindrance is observed in TDHF calculations with the most neutron-rich calcium isotopes which cannot be explained by vibrational couplings. Transfer reactions are also important in these systems due to charge equilibration processes. Conclusions: Despite its impact on the bare potential, the neutron skin is not seen as playing an important role in the fusion dynamics. However, the charge transfer with exotic projectiles could lead to an increase of the Coulomb repulsion between the fragments, suppressing fusion. The effects of transfer and dissipative mechanisms on fusion with exotic nuclei deserve further studies.
\end{abstract}

DOI: 10.1103/PhysRevC.94.024612

\section{INTRODUCTION}

Heavy-ion collision studies rely on a good understanding of the interplay between the structure of the collision partners and reaction mechanisms. This is particularly important at near-barrier energies, where complex quantum effects such as tunneling and coherent coupling between reaction channels are magnified. In particular, these quantum effects have a strong impact on fusion between two nuclei and are highly sensitive to the structure of these nuclei. For instance, a variation of a few neutrons in the choice of the target could lead to variations of sub-barrier fusion cross sections by orders of magnitudes $[1,2]$.

In fact, the discovery that fusion is strongly influenced by the initial structure of the reactants came as a surprise [3,4]. Indeed, the collision partners quickly lose their identity when they merge, on typical time scales of a few zeptoseconds $\left(10^{-21} \mathrm{~s}\right)$. Nevertheless, this time frame is sufficiently long to enable couplings between the relative motion and internal collective excitations $[5,6]$. These couplings lead to a structuredependent distribution of fusion barriers [7], a phenomenon which has been widely studied experimentally (see Refs. [8,9] for reviews) and traditionally interpreted within the coupledchannel framework (see Refs. [8-11] for reviews). Coupling to rotational $[2,12,13]$ and low-lying vibrational $[1,2,5,14,15]$ states, as well as to transfer [4,16-20] and breakup of colliding partners [21-23], have been shown to have a strong impact on fusion.

*kirsten.vo-phuoc@anu.edu.au
This modern picture of heavy-ion fusion has been achieved thanks to high-precision measurements with stable beams. The recent development of exotic beam facilities has now opened new perspectives for fusion studies. In particular, the role of large neutron halos or skins, soft dipole resonances, weak nucleon binding energy, and large isospin asymmetry could be systematically investigated experimentally in the near future. All are expected to impact fusion [24-26]. The first fusion studies with exotic beams focused on reactions with light neutron-rich nuclei to understand the impact of their neutron excess and enhanced breakup and transfer due to weak neutron binding energies [27-31]. More recent experiments have used heavier exotic beams, such as ${ }^{132} \mathrm{Sn}$ and ${ }^{134} \mathrm{Te}$, to study the interplay between transfer and fusion $[32,33]$.

The purpose of this work is to study the fusion mechanism away from stability. We focus on the fusion barrier, $V_{B}$, which is sensitive to the structure of the collision partners. Fusion barriers were systematically studied for stable nuclei as soon as heavy-ion beams with sufficient energies were available $[34,35]$. The barrier is generated by the competition between Coulomb repulsion and strong nuclear attraction between the fragments. Exotic structures, for example, large neutron skins, could affect the barrier radius, $R_{B}$, and in turn $V_{B}$. A legitimate question is also to ask how these effects of nuclear structure on the fusion barrier would be impacted by the reaction dynamics [36].

In order to investigate both static and dynamic effects on fusion with exotic nuclei, we use a microscopic approach based on the Hartree-Fock (HF) mean-field approximation. In this approximation, each nucleon is assumed to move independently in the self-consistent mean-field generated 
by the other nucleons. Static HF calculations account for important nuclear-structure characteristics such as shell effects [37], ground-state deformation [38], and neutron skin [39]. Dynamical effects can also be accounted for in time-dependent Hartree-Fock (TDHF) theory, which allows the nuclear density and thus the mean field to evolve in time. The fact that TDHF calculations treat static and dynamical effects on the same footing is particularly attractive to study the interplay between nuclear structure and reaction mechanisms (see Refs. [40,41] for reviews). Another invaluable feature for exotic systems is that no prior knowledge of the structure of the reactants is required. Indeed, the only parameters are those of the effective interaction between nucleons, usually of the Skyrme type [42]. For instance, time-dependent microscopic calculations have been successful in reproducing fusion reactions with exotic ${ }^{132} \mathrm{Sn}$ beam [43].

We focus our study on collisions of calcium isotopes with ${ }^{116} \mathrm{Sn}$. The static effect of neutron skin on the bare potential barrier is first studied in Sec. II with the frozen-HF method. TDHF calculations are then presented in Sec. III to investigate the net effect of the dynamics on the fusion thresholds for these systems. In order to understand the contribution of the vibrational couplings to the dynamics we perform coupledchannel calculations in Sec. IV B, where the properties of the vibrational states are calculated with a TDHF code using linear response theory. Finally, the importance of transfer channels is investigated with TDHF calculations in Sec. V.

\section{STATIC EFFECTS}

\section{A. The frozen Hartree-Fock approach}

The nucleus-nucleus potential between two ground-state nuclei, otherwise known as the bare potential where the densities of the nuclei stay "frozen" at all points [44], was calculated using the frozen HF method $[45,46]$. One first has to separately compute the HF ground states of the nuclei. Then, the total energy of the system $E(\mathbf{R})$ is computed from the total density by setting a distance vector $\mathbf{R}$ between the centers of mass.

The nuclear part of the nucleus-nucleus potential, denoted by $V$, is given by [44]

$$
V(\mathbf{R})=E(\mathbf{R})-E_{\mathrm{HF}}\left[\rho_{1}\right]-E_{\mathrm{HF}}\left[\rho_{2}\right],
$$

where $\mathbf{R}$ is the position vector between the centers of masses of the two separate systems which have ground-state densities $\rho_{1}$ and $\rho_{2}$ respectively. The total interaction energy can be written as an integral of a local energy density function,

$$
E(\mathbf{R})=\int \mathcal{E}\left[\rho_{1}(\mathbf{r})+\rho_{2}(\mathbf{R}-\mathbf{r})\right] d \mathbf{r} .
$$

The HF energy for each nucleus $(j=1,2)$ reads

$$
E_{\mathrm{HF}}\left[\rho_{j}\right]=\int \mathcal{E}\left[\rho_{j}(\mathbf{r})\right] d \mathbf{r} .
$$

The same Skyrme energy density functional [42] is used to compute both ground-state densities and the nuclear interac- tion between the nuclei. Then, the set of parameters of the Skyrme functional is the only input needed to compute the frozen HF potential. Two parametrizations of the Skyrme interaction, the SLy4d [47] and UNEDF1 [48] parametrizations, have been used for the most part of this work. Both ignore the center-of-mass corrections in the fitting procedure so they are suitable not just for static calculations but also dynamical simulations $[41,48]$.

The HF ground states of the nuclei were calculated using the EV8 [49] code. Pairing correlations were included at the BCS level with a surface pairing interaction [50] with a densitydependent pairing force $[51,52]$ of the form

$$
v_{\text {pair }}\left(\mathbf{r}_{1}, \mathbf{r}_{2}\right)=\tilde{t_{0}} \delta\left(\mathbf{r}_{1}-\mathbf{r}_{2}\right)\left(1-\frac{\rho(\tilde{\mathbf{r}})}{\tilde{\rho}_{0}}\right),
$$

where $\tilde{\mathbf{r}}=\left(\mathbf{r}_{1}+\mathbf{r}_{2}\right) / 2$ and with parameter values $\tilde{t}_{0}=$ $1000 \mathrm{MeV} \mathrm{fm}{ }^{3}$ and $\tilde{\rho}_{0}=0.16 \mathrm{fm}^{-3}$. Pairing correlations are known to have a small effect on fusion [53]. However, they avoid spurious ground-state deformations (by distributing the occupation numbers near the Fermi surface) which could in turn have a spurious effect on the prediction of the fusion barrier.

All nuclei studied here are spherical in their ground state and were calculated on a three-dimensional (3D) grid with cubic box of size $22.4^{3} \mathrm{fm}^{3}$ with three planes of symmetry and with mesh size $\Delta x=0.8 \mathrm{fm}$. All HF calculations henceforth use this same mesh size.

The calculations for the frozen HF bare potential were done on a box size $67.2 \times 22.4 \times 22.4 \mathrm{fm}^{3}$ in the $x-y-z$ orientation where the collision axis is the $x$ axis. The plane $z=0$ is a plane of symmetry. The maximum distance between the two centers of mass was $44.8 \mathrm{fm}$.

The resulting nucleus-nucleus potential obtained from the sum of the nuclear part (1) and the Coulomb potential is shown in Fig. 1 for the sample system ${ }^{40} \mathrm{Ca}+{ }^{116} \mathrm{Sn}$. The

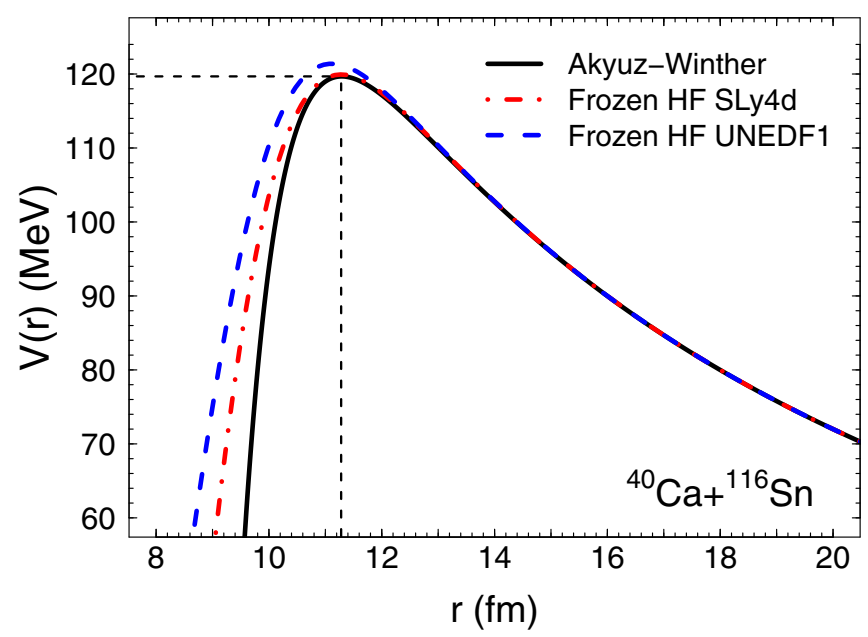

FIG. 1. Example of the bare nucleus-nucleus potential from the frozen HF method (dashed and dot-dashed lines) and the AkyüzWinther phenomenological potential [54] (solid line) for ${ }^{40} \mathrm{Ca}+{ }^{116} \mathrm{Sn}$. The dashed lines show the maximum AW barrier energy at $V=119.7 \mathrm{MeV}$ and $r=11.3 \mathrm{fm}$. 
phenomenological Akyüz-Winther (AW) [54] nucleus-nucleus potential is also shown for comparison.

The agreement between the fully microscopic frozen HF approach and the phenomenological potential in terms of height and position of the barrier is remarkable. Both approaches disagree, however, on the inner part of the potential. There is naturally a large uncertainty in predicting the form of the potential in macroscopic phenomenological approaches when the two nuclei strongly overlap $[55,56]$. The frozen HF approach, however, does not rely on an a priori guess of the form of the potential, and the microscopic calculations predict a wider barrier than the AW potential. Note that the present frozen HF calculations naturally incorporate effects from incompressibility [56] but neglect the Pauli exclusion principle between nucleons belonging to different nuclei. The latter is expected to reduce the attraction between the nuclei inside the barrier and then to further increase the barrier width. The inner part of the potential affects deep sub-barrier fusion cross sections $[57,58]$ but it is not expected to have a large impact on near-barrier data from which the fusion barrier distributions [7] are extracted experimentally [2,8]. Therefore, a modification of the inner part of the potential would not affect the conclusions of this work.

\section{B. Results}

The bare potentials in ${ }^{A} \mathrm{Ca}+{ }^{116} \mathrm{Sn}$ systems have been computed with the frozen HF approach for the SLy4d and UNEDF1 parametrizations of the Skyrme interaction. The resulting barrier energies, $V_{B}$, are presented in Fig. 2 together with fusion barriers obtained from the AW potential.

A reduction of the barrier height is observed with increasing $A$ in each set of calculations due to the increase in size of the calcium isotopes (see Fig. 3). However, the HF calculations for both Skyrme parametrizations also exhibit a faster reduction of $V_{B}$ with increasing $A$ for $A \geqslant 48$. This feature is not seen with the AW phenomenological potential, which is a simple smooth function of the mass number $A$ and does not account for quantum shell effects.

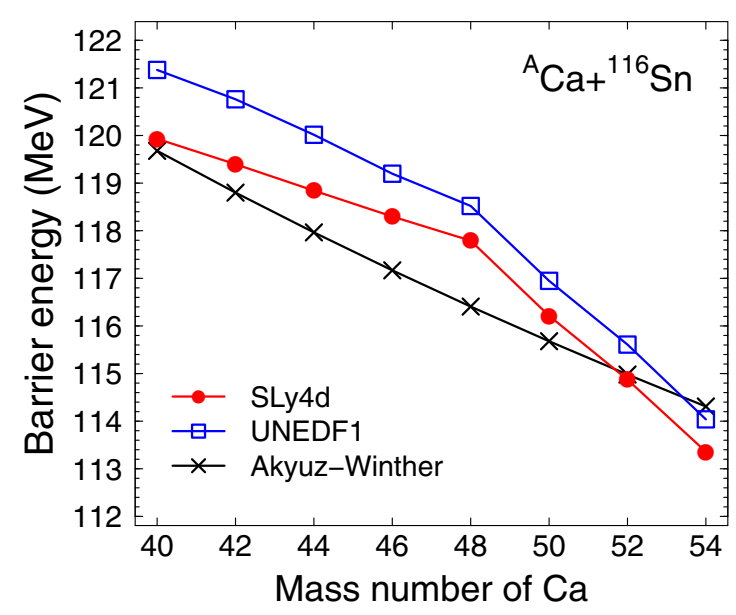

FIG. 2. Frozen HF barriers of calcium isotopes on ${ }^{116} \mathrm{Sn}$. Two different parametrizations are used. Also included are the macroscopic Akyüz-Winther potential barriers.

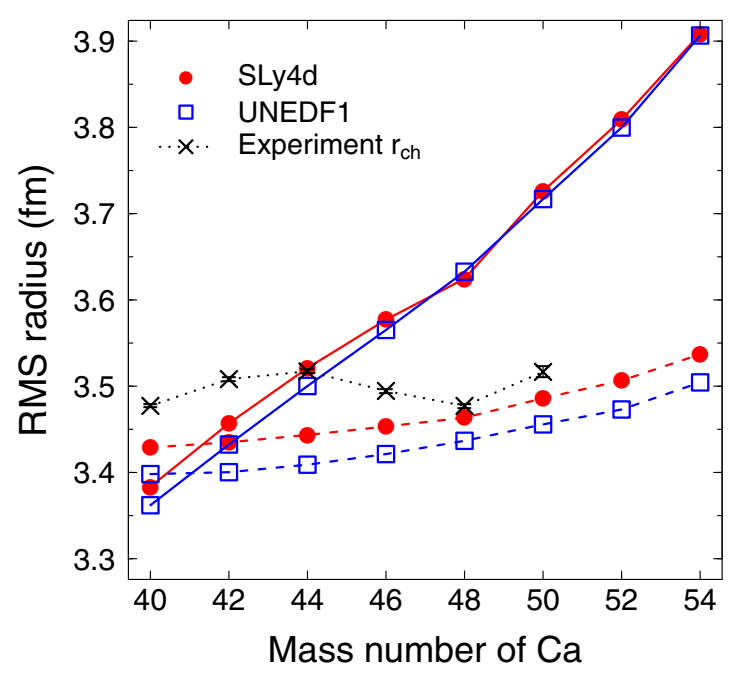

FIG. 3. HF proton (dashed lines) and neutron (solid lines) root mean square radii in calcium isotopes for two parametrizations of the Skyrme functional. The experimental charge radii (crosses) are from Refs. [59,60].

In order to interpret this change of behavior near the doubly magic ${ }^{48} \mathrm{Ca}$ isotope in the microscopic calculations, the root-mean-square (rms) radii, both proton and neutron, have been computed for the calcium HF ground states. These are shown in Fig. 3. There is a change of gradient in the neutron rms radius at the ${ }^{48} \mathrm{Ca}$ nucleus in both parametrizations, indicating the development of a neutron skin. A more rapid increase of rms radius after ${ }^{48} \mathrm{Ca}$ means a more rapid decrease of barrier energies due to the lowering of the Coulomb repulsion between the reactants. Also included in Fig. 3 are the experimental charge rms radii $[59,60]$. These values are relatively close to the calculated proton radii, especially for the doubly magic nuclei. The deviations are larger for midshell nuclei due to correlations not accounted for at the mean-field level [61].

The neutron single-particle shell levels arising from the HF ground states of the calcium nuclei help us understand why the HF rms radius behaves this way. Between ${ }^{40} \mathrm{Ca}$ and ${ }^{48} \mathrm{Ca}$ the $1 f_{7 / 2}$ neutron shell is progressively filled. Then, after ${ }^{48} \mathrm{Ca}$ there is a magic shell gap of approximately $5 \mathrm{MeV}$ in energy until the next $2 p_{3 / 2}$ shell. The last occupied shell in ${ }^{54} \mathrm{Ca}$ is the $2 p_{1 / 2}$ shell. The $2 p$ neutron levels are weakly bound in these calcium isotopes and, in comparison to $1 f$ levels, they also have an additional node. These effects can explain the faster increase of the neutron radius with $A$ for isotopes heavier than ${ }^{48} \mathrm{Ca}$.

To conclude, the development of a neutron skin in exotic calcium isotopes could significantly lower the barrier. This phenomenon is due to quantum shell effects and is not accounted for in standard parametrizations of the nucleusnucleus potential. The latter are then only valid close to the valley of stability. The lowering of the barrier due to the neutron skin is purely a static effect and it remains to investigate how this effect is affected by the dynamics. 


\section{DYNAMIC EFFECTS}

\section{A. The time-dependent Hartree-Fock approach}

Obtaining a barrier energy for fusion using frozen groundstate densities is a useful starting point but a more realistic barrier energy naturally should include the dynamics of the nuclei as they approach each other, for example, the possibility of vibrations, rotations, and transfer. Traditionally this is done within the coupled-channel formalism. An alternative approach is to use the time-dependent Hartree-Fock (TDHF) method. Early TDHF applications already included vibration [62], fission [63], and reaction [64] studies. However, realistic calculations, including all dynamics at the mean-field level, have only been made possible in the past decade thanks to the development of three-dimensional TDHF codes [47,65-68] including spin-orbit couplings [69]. In particular, the TDHF approach has been shown to give different fusion thresholds than the corresponding frozen HF bare potential computed with the same functional, indicating an important role of dynamics on the fusion mechanism [46,70-73].

The TDHF equation reads

$$
i \hbar \frac{d}{d t} \rho=[h[\rho], \rho]
$$

where $\rho$ is the one-body density matrix and $h[\rho]$ is the selfconsistent single-particle HF Hamiltonian. TDHF codes solve this equation in the canonical basis in which the elements of the one-body density matrix read

$$
\rho\left(\mathbf{r}, s, q ; \mathbf{r}^{\prime}, s^{\prime}, q^{\prime}\right)=\sum_{i} n_{i} \varphi_{i}(\mathbf{r}, s, q) \varphi_{i}^{*}\left(\mathbf{r}^{\prime}, s^{\prime}, q^{\prime}\right),
$$

where $\varphi_{i}$ are the single-particle wave functions with occupation numbers $n_{i}$ and $\mathbf{r}, s$, and $q$ denote position, spin, and isospin, respectively. In this basis, the TDHF equation can be written as a set of nonlinear Schrödinger equations for each single-particle wave function

$$
i \hbar \frac{d}{d t} \varphi_{i}=h[\rho] \varphi_{i} .
$$

This set of equations is solved iteratively in time, with the HF Hamiltonian determined at every iteration according to its relationship with the energy density functional

$$
h[\rho]\left(\mathbf{r}, s, q ; \mathbf{r}^{\prime}, s^{\prime}, q^{\prime}\right)=\frac{\delta E[\rho]}{\delta \rho\left(\mathbf{r}^{\prime}, s^{\prime}, q^{\prime} ; \mathbf{r}, s, q\right)} .
$$

The effects of dynamics on fusion were studied here with the TDHF3D code [47]. The calculations start with the HF ground states of the nuclei put together in a larger box as done for frozen HF calculations. Now, a mean field for the entire system is generated by all the independent particles from both nuclei. A Galilean boost is applied to each nucleus at initial time $t=0$ and the mean field evolves self-consistently [64]. The evolution of the occupied single-particle wave functions of the nuclei with respect to time is computed as the nuclei move relative to each other.

The occupation numbers $n_{i}$ are those determined in the ground-state static calculations by the pairing interaction (2) in the BCS approximation. These occupation numbers are kept constant during the dynamics, that is, we used the so- called frozen occupation number approximation [65]. A more sophisticated approach would imply a BCS [74] treatment to allow the occupation numbers $n_{i}$ to evolve in time. Unlike in fission in which dynamical pairing correlations play an important role $[63,75,76]$, they have been shown to barely affect fusion [53] and are neglected in the present work.

The TDHF method is much more computationally demanding than its static counterpart. As for the frozen case, a plane of symmetry at $z=0$ is assumed in the TDHF3D code to speed up numerical simulations. All calculations were done using the same box conditions as for the frozen HF calculations and with both the SLy4d and UNEDF1 parametrizations of the Skyrme functional. The starting distance must be large enough so that Coulomb excitation is properly accounted for in the approach phase. This is particularly important for calculations involving large charge products $Z_{1} Z_{2}$ [77,78]. Starting at a separation distance between the centers of masses of $44.8 \mathrm{fm}$ is large enough to account for this effect in $\mathrm{Ca}+\mathrm{Sn}$ collisions. The time-step size used between each iteration was $1.5 \times 10^{-24} \mathrm{~s}$ to ensure convergence. This study focuses on $L=0$ angular momentum fusion barriers; therefore only central collisions are considered.

To extract a fusion barrier energy from the TDHF method, the distance between center of masses of the two interacting nuclei was used. The notion of defining separate centers of mass in a single mean field (for the whole system) is addressed by defining a neck plane perpendicular to the collision axis. At each time, its purpose is to separate the entire density of the system into two fragments [71]. Using this, the centers of masses of these two fragments and the distance between them can then be calculated at each time step. Fusion for the system was deemed successful if, at a given center of mass energy $E_{\text {c.m. }}$, the distance between the centers of masses remained below $\sim 10 \mathrm{fm}$ after $4.5 \mathrm{zs}$.

An example of the distance between center of mass versus time is given in Fig. 4 for ${ }^{40} \mathrm{Ca}+{ }^{116} \mathrm{Sn}$. The solid line is associated with a trajectory where fusion occurred. The TDHF fusion probability for a given initial condition is either 0 or 1 as there is no tunneling of the many-body wave function taken into account. In TDHF calculations, the fusion barrier energy is really then a fusion threshold. This threshold is found by varying $E_{\text {c.m. }}$ in steps of $0.1 \mathrm{MeV}$. Each TDHF fusion threshold energy then has a numerical uncertainty of $\pm 0.05 \mathrm{MeV}$.

\section{B. TDHF results}

The TDHF fusion thresholds are plotted with the corresponding frozen HF fusion barriers in Fig. 5 for the SLy4d and UNEDF1 parametrizations. In both parametrizations, we can see that for the reactions with ${ }^{40-50} \mathrm{Ca}$ projectiles, including dynamics has lowered the fusion barrier overall. For all systems it is noticeable that dynamic effects override the static effects seen in the frozen HF barriers as there is no longer a change of slope in $V_{B}(A)$ near ${ }^{48} \mathrm{Ca}$. Importantly, this means that the sub-barrier fusion enhancement expected from the neutron skin in a static picture is in fact not present when dynamic processes are included.

For SLy4d, the fusion barrier is actually increased by the dynamics for ${ }^{52,54} \mathrm{Ca}$ projectiles. This is surprising, as it is 


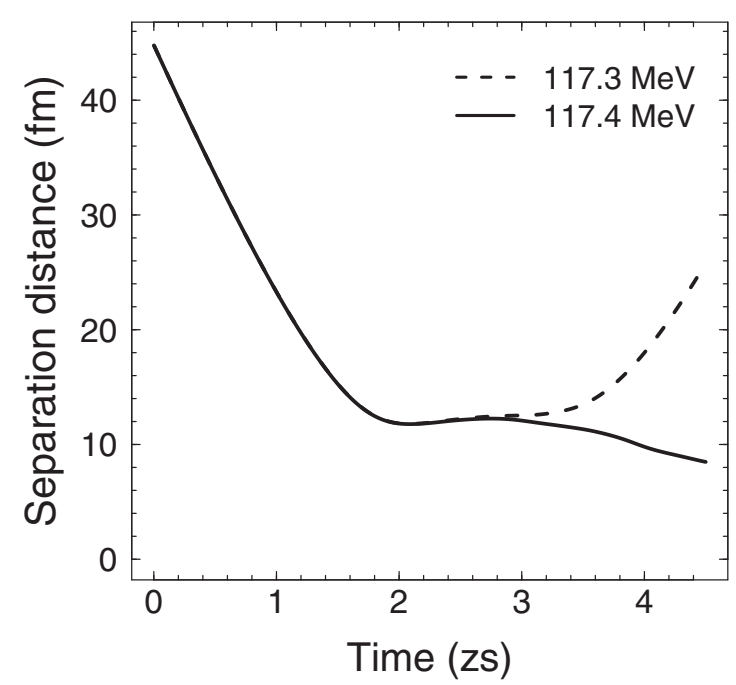

FIG. 4. Distance between fragment centers of masses in ${ }^{40} \mathrm{Ca}+{ }^{116} \mathrm{Sn}$ central collisions as a function of time. Fusion is achieved at $E_{\text {c.m. }}=117.4 \mathrm{MeV}$ (solid line) while separation of the nuclei occurs at $E_{\text {c.m. }}=117.3 \mathrm{MeV}$ (dashed line).

expected that dynamics should in general lower the fusion barrier [11]. For UNEDF1, the fusion barrier has been lowered in all cases, however, with a much smaller magnitude for the most exotic calcium isotopes. Therefore, both interactions predict that for the most neutron-rich isotopes a dynamical mechanism occurs which counterbalances the usual lowering of fusion thresholds due to couplings.

TDHF calculations intrinsically incorporate a wide variety of dynamical effects, such as couplings to vibration and transfer channels. It is therefore desirable to investigate how individual dynamical effects modify the fusion barrier. This question is addressed in the next two sections.

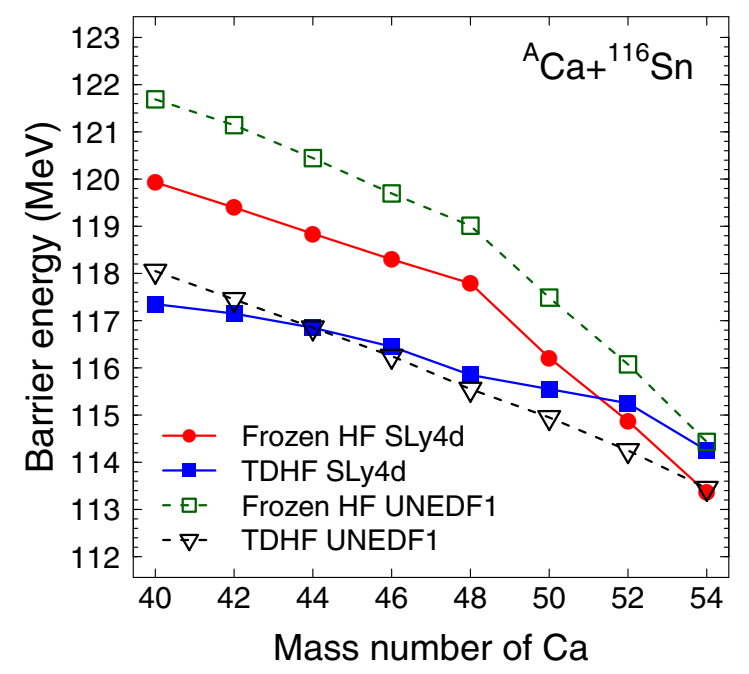

FIG. 5. Bare potential barrier energies from the frozen HF method and TDHF fusion thresholds for ${ }^{A} \mathrm{Ca}+{ }^{116} \mathrm{Sn}$ are plotted with respect to the calcium mass number for the SLy4d (solid lines) and UNEDF1 (dashed lines) parametrizations.

\section{VIBRATIONAL COUPLINGS}

Our aim in this section is to investigate the effect of vibrational couplings on the fusion barrier. The TDHF approach includes all types of dynamical couplings, but only at the mean-field level, and without the possibility to disentangle each contribution in a straightforward way. Therefore, we use a method based on a comparison between standard TDHF and coupled-channel calculations with frozen HF and explicitly including particular dynamical modes for example vibrations, developed in Ref. [46], to investigate the importance of low-lying vibrations on the fusion barrier. The inputs to enable couplings to the collective states are obtained from TDHF.

\section{A. Nuclear vibrations}

Coupled-channel calculations require knowledge of the energy of the collective states as well as their transition strengths. Both quantities can be obtained from TDHF calculations of a single nucleus [62]. This method has been widely applied to study giant-resonances $[65-67,74,79,80]$ but more rarely to low-lying vibrations $[46,72,74]$. Although TDHF calculations can be used to study nonlinear vibrations [81,82], the extraction of the transition strength relies on the linear regime, in which case it is equivalent to the random-phase approximation (RPA). Note that TDHF in coordinate space allows for particle evaporation $[83,84]$ and thus the escape width is included. The spreading width, however, involves two-body mechanisms not accounted for in TDHF. As before, only the initial static pairing correlations are included.

A basic outline of linear response theory follows. Let us consider a multipole moment $\hat{Q}_{\lambda}$ of multipolarity $\lambda$. The transition amplitude between the ground state $|0\rangle$ with energy $E_{0}$ and the excited state $|v\rangle$ with energy $E_{v}$ is defined as $q_{v}=\left\langle v\left|\hat{Q}_{\lambda}\right| 0\right\rangle$. In order to calculate the transition probability $\left|q_{v}\right|^{2}$, a small excitation is applied on the nucleus at the initial time with a boost of the form

$$
|\Psi(0)\rangle=e^{-i \varepsilon \hat{Q}_{\lambda}}|0\rangle,
$$

where $\varepsilon$ is the boost velocity and quantifies the intensity of the excitation. The boost induces an oscillation of the multipole moment expectation value which is given by

$$
\left\langle\hat{Q}_{\lambda}\right\rangle(t)=-2 \varepsilon \sum_{\nu}\left|q_{\nu}\right|^{2} \sin \left[\left(E_{v}-E_{0}\right) t / \hbar\right]+O(\varepsilon) .
$$

The linear regime is obtained by choosing $\varepsilon$ small enough so that $\left\langle\hat{Q}_{\lambda}\right\rangle$ is linearly proportional to $\varepsilon$. The strength function is then computed from a sine Fourier transform of $\left\langle\hat{Q}_{\lambda}\right\rangle(t)$

$$
\begin{aligned}
Q_{\lambda}(E) & =\lim _{\varepsilon \rightarrow 0} \frac{-1}{\pi \hbar \varepsilon} \int_{0}^{\infty} d t\left\langle\hat{Q}_{\lambda}\right\rangle(t) \sin (E t / \hbar) \\
& \simeq \sum_{\nu}\left|q_{\nu}\right|^{2} \delta\left[E-\left(E_{\nu}-E_{0}\right)\right] .
\end{aligned}
$$

In practice, $\left\langle\hat{Q}_{\lambda}\right\rangle(t)$ is only computed over a finite time. To avoid spurious oscillations in the strength function, $\left\langle\hat{Q}_{\lambda}\right\rangle(t)$ is filtered in the time domain by multiplying it by a scaled half-Gaussian damping function reaching zero at the end of the calculation [65]. This damping function adds only a small width to the peaks in the strength function. 

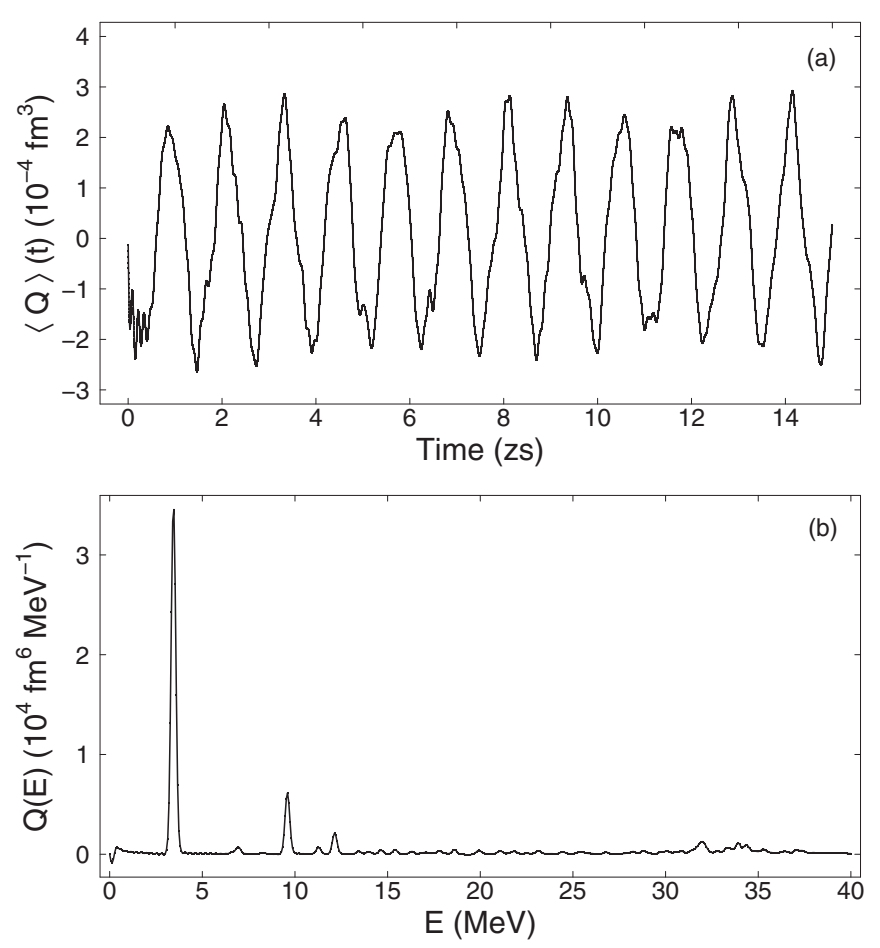

FIG. 6. (a) Time evolution of the octupole moment induced by an octupole boost on ${ }^{40} \mathrm{Ca}$ in the linear regime. (b) Associated strength function computed from Eq. (5).

We focus on quadrupole and octupole vibrations which can strongly couple to the relative motion. The quadrupole and octupole operators are defined as

$$
\begin{aligned}
& \hat{Q}_{2}=\sqrt{\frac{5}{16 \pi}} \sum_{i=1}^{A}\left(2 \hat{x}^{2}-\hat{y}^{2}-\hat{z}^{2}\right), \\
& \hat{Q}_{3}=\sqrt{\frac{7}{16 \pi}} \sum_{i=1}^{A}\left[2 \hat{x}^{3}-3 \hat{x}\left(\hat{y}^{2}+\hat{z}^{2}\right)\right],
\end{aligned}
$$

respectively. The operators $\hat{x}, \hat{y}$, and $\hat{z}$ are single-particle observables and the sums run over each nucleon.

All TDHF vibration calculations were performed using the SLy4d interaction in the same box size as for HF ground states in Sec. II but with one plane of symmetry $z=0$ and for a total time of $15 \mathrm{zs}$. An example of evolution of the octupole moment following an octupole boost on ${ }^{40} \mathrm{Ca}$ is presented in Fig. 6(a). A strong oscillation is observed, producing an intense peak at $3.44 \mathrm{MeV}$ in the strength function plotted in Fig. 6(b). This peak is associated to the $3_{1}^{-}$first phonon of the low-lying collective octupole mode. The transition probability $\left|q_{v}\right|^{2}$ is obtained from the area of the peak and transformed into a deformation parameter according to

$$
\beta_{\lambda}=\frac{4 \pi\left|q_{\nu}\right|}{3 A R_{0}^{\lambda}}
$$

The radius $R_{0}$ is taken as the radius of the isodensity surface at half the saturation density $\rho_{0} / 2=0.08 \mathrm{fm}^{-3}$.

The low-lying octupole phonon energies and their associated deformation parameters computed with TDHF are re-
TABLE I. TDHF and experimental [85] excitation energies and deformation parameters for $3_{1}^{-}$states in calcium isotopes.

\begin{tabular}{lcccc}
\hline \hline$A(\mathrm{Ca})$ & \multicolumn{2}{c}{ TDHF } & \multicolumn{2}{c}{ Experiment } \\
\hline & $E_{3}$ & $\beta_{3}$ & $E_{3}$ & $\beta_{3}$ \\
40 & 3.44 & 0.224 & 3.737 & $0.30-0.41$ \\
42 & 4.14 & 0.195 & 3.447 & 0.26 \\
44 & 4.68 & 0.165 & 3.308 & $0.23-0.26$ \\
46 & 5.14 & 0.141 & 3.614 & 0.16 \\
48 & 5.52 & 0.109 & 4.507 & $0.17-0.25$ \\
50 & 4.62 & 0.168 & & \\
52 & 3.48 & 0.221 & & \\
54 & 2.92 & 0.226 & & \\
\hline \hline
\end{tabular}

ported in Table I alongside experimental values. The purpose of this comparison is not to achieve the best possible description of vibrational modes (we leave that for a future work) but rather to verify what types of vibrational couplings are included in the TDHF dynamics. The TDHF results in Table I can be interpreted in a simple spherical shell-model picture. The $3_{1}^{-}$state in ${ }^{40} \mathrm{Ca}$ is a coherent sum of one-particle-one-hole excitations across the $N=Z=20$ magic shell gap. Adding neutrons to the $1 f_{7 / 2}$ shell blocks neutrons excitations across the $N=20$ magic gap, hindering collectivity. This is seen as a reduction of $\beta_{3}$ going from $A=40$ to 48 and results in an increase of $E_{3}$ due to less residual interaction. There is also a large energy gap between $1 f_{7 / 2}$ and the next positive parity level $\left(1 g_{9 / 2}\right)$. Having filled the $1 f_{7 / 2}$ shell, collectivity then increases due to increased excitations between the $f p$ shell and $1 g_{9 / 2}$ for $A>48$.

The above observations are in qualitative agreement with the experimental data for doubly magic nuclei $\left({ }^{40,48} \mathrm{Ca}\right)$. The situation is more complicated for midshell nuclei, which could be affected by pairing correlations neglected in the RPA residual interaction. However, the energies agree to better than $30 \%$ and the deformation parameters, while underestimated in TDHF, are of the same order of magnitude. This is sufficient for the purpose of investigating the impact of low-lying octupole vibrations in fusion.

Similar calculations have been performed for quadrupole vibrations. No low-lying collective oscillations are found in ${ }^{40} \mathrm{Ca}$ as $2^{+}$one-particle-one-hole states require excitations across two magic gaps (magic numbers 20 and 28), therefore contributing only to the giant quadrupole resonance. Neutrons in the partially filled $1 f_{7 / 2}$ shell can produce low-lying $2^{+}$ states by breaking a pair and coupling within the same shell. Experimentally, this leads to $2_{1}^{+}$states at $E_{2} \simeq 1-1.5 \mathrm{MeV}$ in ${ }^{42,44,46} \mathrm{Ca}$ isotopes. However, these states have zero excitation energy in TDHF as pairing is neglected. The first $2^{+}$excitations in the strength functions calculated with TDHF for $40<$ $A \leqslant 48$ calcium isotopes are then obtained by promoting $1 f_{7 / 2}$ neutrons across the $N=28$ gap. For the $48<A \leqslant 54$ calcium isotopes also studied here, low-lying $2^{+}$states can be formed by one-particle-one-hole excitations in the $f p$ shell. Overall, we found that these low-lying quadrupole vibrations, as calculated in TDHF, are globally much less collective than the octupole modes. The largest quadrupole deformation 
TABLE II. Woods-Saxon fit parameters for frozen HF nucleusnucleus potential of ${ }^{A} \mathrm{Ca}+{ }^{116} \mathrm{Sn}$ reactions with the SLy4d interaction.

\begin{tabular}{lccc}
\hline \hline$A(\mathrm{Ca})$ & $V_{0}(\mathrm{MeV})$ & $R(\mathrm{fm})$ & $a(\mathrm{fm})$ \\
\hline 40 & 76.433 & 1.199 & 0.611 \\
42 & 72.773 & 1.202 & 0.604 \\
44 & 75.171 & 1.198 & 0.603 \\
46 & 73.054 & 1.199 & 0.600 \\
48 & 75.254 & 1.195 & 0.599 \\
50 & 90.939 & 1.179 & 0.641 \\
52 & 102.237 & 1.170 & 0.667 \\
54 & 125.215 & 1.153 & 0.701 \\
\hline \hline
\end{tabular}

parameter is found in the ${ }^{48} \mathrm{Ca} 2_{1}^{+}$state at $E_{2}=3.828 \mathrm{MeV}$ with $\beta_{2}=0.078$, in good agreement with experiment $\left(E_{2}=\right.$ $3.832 \mathrm{MeV}$ and $\beta_{2}=0.101 \pm 0.017$ [86]). We have checked that, in TDHF, the main contribution of coupling effects on the barrier comes from the octupole states and we therefore only include $3_{1}^{-}$states in the following coupled-channel calculations.

\section{B. Coupled-channels calculations}

Coupled channel (CC) calculations of ${ }^{A} \mathrm{Ca}+{ }^{116} \mathrm{Sn}$ have been performed with the CCFULL+ code [87]. The nuclear potential is taken to be in the Woods-Saxon form,

$$
V_{N}(r)=\frac{-V_{0}}{1+\exp [(r-R) / a]},
$$

with the usual three parameters of potential depth $V_{0}$, diffuseness $a$, and radius $R$. In all calculations these parameters were taken from fitting the Woods-Saxon potential to the frozen HF bare potential obtained with the SLy4d interaction to reproduce the barrier energy within a $1-\mathrm{keV}$ error. The values of the Woods-Saxon parameters are given in Table II. The energy and deformation parameter of the $3_{1}^{-}$state were taken from the TDHF results in Table I. No transfer coupling was included in any of the $\mathrm{CC}$ calculations.

The barrier distribution $D(E)$ has been calculated from the CCFULL fusion cross section using [7]

$$
D(E)=\frac{d^{2}(E \sigma)}{d E^{2}} .
$$

The barrier distribution is positive for energies ranging from $0 \mathrm{MeV}$ up until some particular energy $E^{\prime}$, above for which it becomes negative [8]. The average fusion barrier is then calculated using the centroid of $D(E)$ with the upper integration limit of $E$ being $E^{\prime}$,

$$
V_{B}=\frac{\int_{0}^{E^{\prime}} E D(E) d E}{\int_{0}^{E^{\prime}} D(E) d E} .
$$

We have checked that, when no couplings are included in the CCFULL calculations, the resulting centroid of the barrier distribution $D(E)$ matches the frozen-HF barrier. Inclusion of coupling to the first octupole phonon in the calcium isotopes systematically reduces the centroid of $D(E)$ by up to $\sim 1.5 \mathrm{MeV}$, as shown in Fig. 7 .

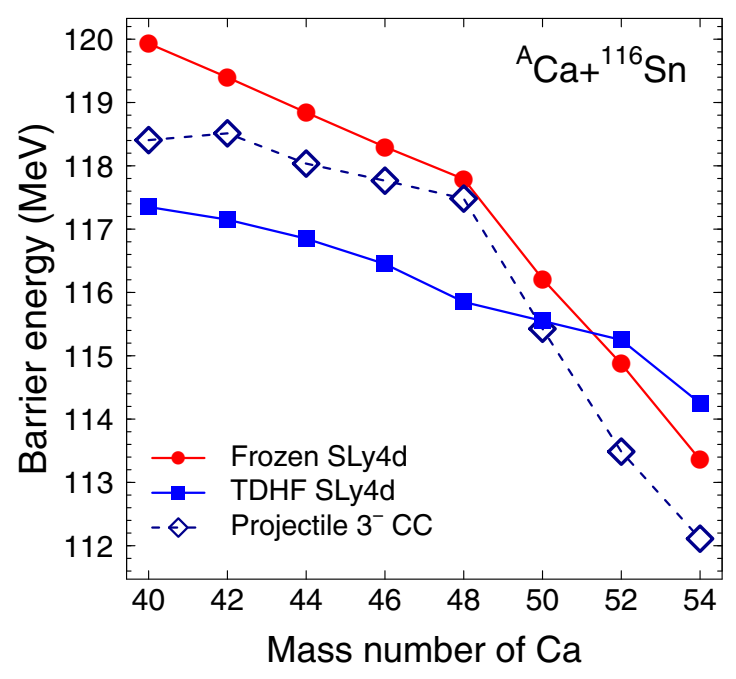

FIG. 7. Frozen HF (cirlces) barriers in ${ }^{A} \mathrm{Ca}+{ }^{116} \mathrm{Sn}$ are compared with TDHF fusion thresholds (squares) and coupled-channels average barriers with couplings to the $3_{1}^{-}$state in calcium isotopes (diamonds).

The impact of coupling to low-lying quadrupole phonons in calcium isotopes has also been studied using experimental data on the $2_{1}^{+}$state (when available). Although this coupling may modify the shape of the barrier distribution, it barely affects its centroid. Higher energy states, such as giant quadrupole resonances, have only a small impact on the barrier $[46,88]$. Coupling to the $3_{1}^{-}$state in ${ }^{116} \mathrm{Sn}$ further reduces the barrier for the systems ${ }^{42-54} \mathrm{Ca}+{ }^{116} \mathrm{Sn}$ by less than $0.2 \mathrm{MeV}$.

The CC calculations confirm that the vibrational states included in the TDHF calculations lower the fusion threshold. This helps explain the lowering of the barrier due to dynamical effects as observed in Fig. 5 for reactions with the nonexotic calcium isotopes. However, vibrational couplings cannot explain why the fusion threshold is actually higher than the frozen HF barrier barrier (with SLy4d) for the most exotic nuclei.

\section{ROLE OF TRANSFER}

While vibrational couplings usually lower the barrier, the effect of transfer channels is less clear despite several experimental investigations [4,16-20,89,90]. One problem is the difficulty to incorporate transfer channels in coupled-channel calculations [91-94]. Alternatively, microscopic approaches can also be used to study transfer reaction mechanisms in heavy-ion collisions [95-97]. Here, our study of transfer channels is motivated by the observation of an increase of the fusion barrier in ${ }^{52,54} \mathrm{Ca}+{ }^{116} \mathrm{Sn}$ in TDHF calculations and which cannot be explained by vibrational couplings (see Fig. 7).

Transfer probabilities are computed using the particle number projection technique developed in Ref. [96] for systems without pairing, and extended in Ref. [98] for superfluid systems. This method has been used to study multinucleon transfer reactions [96,98-102] and fission [75,103]. Here we use it to determine proton transfer probabilities in ${ }^{A} \mathrm{Ca}+{ }^{116} \mathrm{Sn}$. As the fragments both have magic proton numbers, the 


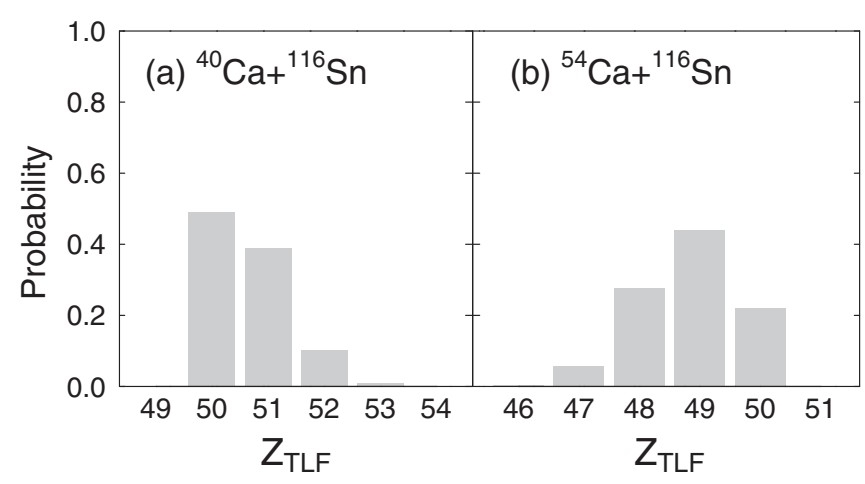

FIG. 8. Proton number probability distributions in the outgoing TLF in ${ }^{40} \mathrm{Ca}+{ }^{116} \mathrm{Sn}$ (a) and ${ }^{54} \mathrm{Ca}+{ }^{116} \mathrm{Sn}$ (b) central collisions at an energy of $99.9 \%$ of the TDHF fusion threshold.

resulting proton transfer probabilities are not affected by pairing correlations so we use the simple projection technique [96].

All calculations were again made using the SLy4d interaction. The probability distribution of the final proton number in the targetlike fragment (TLF) is shown in Fig. 8(a) for ${ }^{40} \mathrm{Ca}+{ }^{116} \mathrm{Sn}$ and in Fig. 8(b) for ${ }^{54} \mathrm{Ca}+{ }^{116} \mathrm{Sn}$ at an energy of $99.9 \%$ of the TDHF fusion threshold. As seen in Fig. 8(a), protons are transferred from the light fragment to the TLF in ${ }^{40} \mathrm{Ca}+{ }^{116} \mathrm{Sn}$ with a probability of $\sim 50 \%$. Conversely Fig. 8(b) shows that ${ }^{116} \mathrm{Sn}$ loses protons in ${ }^{54} \mathrm{Ca}+{ }^{116} \mathrm{Sn}$, with only $\sim 20 \%$ chance to find a tin fragment in the exit channel.

A signature of transfer reactions can also be obtained from the average of the number of nucleons in the final fragments, which is simply determined by integrating the proton and neutron densities around one fragment in the exit channel. Figure 9 (a) shows both the average proton $\left(\bar{Z}_{\mathrm{TLF}}\right)$ and neutron $\left(\bar{N}_{\mathrm{TLF}}\right)$ numbers in the TLF. We observe that $\bar{Z}_{\mathrm{TLF}}$ decreases

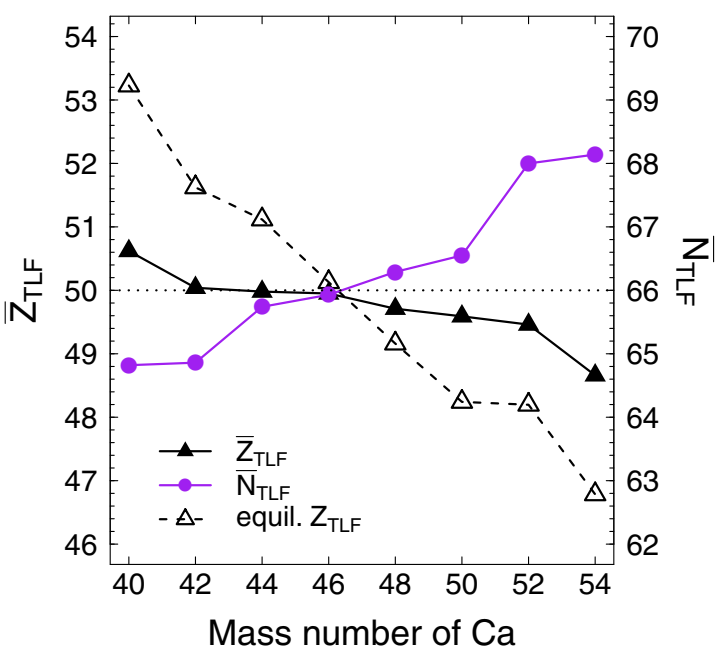

FIG. 9. The average proton (left axis) and neutron number (right axis) of the TLF. The dashed line and open triangles show the anticipated $\bar{Z}_{\mathrm{TLF}}$ value assuming the system is fully equilibriated with the TLF having $N_{\text {TLF }}=\bar{N}_{\text {TLF }}$. The original target $Z$ and $N$ (horizontal dotted line) are also shown. while $\bar{N}_{\text {TLF }}$ increases with increasing calcium mass number, confirming the results in Fig. 8.

The direction of the transfer is determined by a charge equilibration process where the initial neutron-to-proton ratio $N / Z$ asymmetry between the fragments is reduced after contact. This is a manifestation of positive $Q$ values for transfer reactions induced by the symmetry energy, studied in detail with TDHF in transfer reactions [99,104]. The dashed line in Fig. 9 shows the equilibriated numbers of protons for the given $\bar{N}$, assuming that the projectile and target both have the $N / Z$ of the compound system. This line indicates that, at this collision energy, the transfer reactions do not fully equilibriate the reactants. However, it should be regarded as an upper limit since the equilibration acts to increase binding rather than truly equilibriate the neutron-to-proton ratio.

The present calculations indicate that neutron transfer is stronger than proton transfer in this process, resulting in net mass transfer to the light calcium isotopes and from the heavier isotopes. As shown in Fig. 3, the rms radii of the neutrons in the calcium isotopes are generally larger than those for the protons, making them more accessible for transfer. The influence of neutron transfers on fusion is not fully understood.

When the proton transfer to calcium occurs, the charge product of the fragments increases, which in turn increases the Coulomb repulsion and thus the fusion barrier. This suggests a possible mechanism for the increase of the fusion threshold due to dynamical effects in ${ }^{52,54} \mathrm{Ca}+{ }^{116} \mathrm{Sn}$.

An alternative explanation would be that dissipation of the initial kinetic energy is faster (meaning it occurs at larger distances) with calcium isotopes with $A>48$ due to a larger level density near the Fermi level and weak neutron binding. Further studies are required to better understand the role of transfer and dissipation in the dynamical effects on the fusion barrier. For instance, a simple proxy to the dissipation can be obtained in TDHF from the total kinetic energy loss [105] and from the number of emitted nucleons [106]. More advanced techniques to extract the energy dissipated into excitation energies include a macroscopic reduction procedure [107], the density-constrained TDHF approach [108], and a more general application of the particle number projection technique [109].

\section{CONCLUSION}

A systematic study of fusion barriers in reactions between a stable target $\left({ }^{116} \mathrm{Sn}\right)$ and a chain of calcium projectiles ranging from stable to unstable neutron-rich isotopes has been performed using microscopic approaches based on the Hartree-Fock mean-field approximation.

The bare potential barriers obtained assuming frozen ground-state densities decrease with the calcium mass number. The results also show that the development of a neutron skin for calcium isotopes heavier than ${ }^{48} \mathrm{Ca}$ further decreases the bare barrier.

However, this static effect on the bare barrier disappears when dynamic effects are taken into account via the TDHF approach. The inclusion of dynamical effects globally lowers the fusion threshold except for reactions with the most exotic calcium isotopes studied here $\left({ }^{52,54} \mathrm{Ca}\right)$. Depending on the 
choice of the Skyrme interaction, the fusion threshold can even become higher than the bare barrier for these exotic projectiles.

Coupled-channels calculations have been performed to understand the contribution of couplings to low-lying vibrations to the dynamical modification of the barrier. The results show that vibrational couplings systematically lower the average barrier and are thus not responsible for the increase of barrier energy, which will hinder fusion with exotic calcium projectiles.

The importance of transfer channels near the barrier has also been investigated with TDHF calculations for these systems. The results, which can be interpreted in terms of a simple charge equilibration process, suggest that the Coulomb repulsion is increased due to charge transfer in ${ }^{52,54} \mathrm{Ca}+{ }^{116} \mathrm{Sn}$. This mechanism provides a possible explanation for the fusion hindrance in these systems. An increase of dissipation associated with a weakly bound collision partner could also provide a mechanism for fusion hindrance.

More work is needed to get deeper insight into the role of transfer and dissipation mechanisms in microscopic dynamics. It is also desirable to include dynamic pairing correlations as this would affect inelastic excitations and multinucleon transfer probabilities. Various Skyrme parametrizations and effective interactions should also be tested as they may lead to different predictions for reactions with exotic beams. For instance, reactions with the Gogny interaction have recently been performed [110]. The use of effective interactions from the quark-meson coupling model has also shown promising results in static HF calculations of nuclear structure [111] and could be implemented in TDHF codes.

\section{ACKNOWLEDGMENTS}

The authors are grateful to M. Dasgupta, D. J. Hinde, and A. S. Umar for stimulating discussions during this work. This research was undertaken with the assistance of resources from the National Computational Infrastructure (NCI), which is supported by the Australian Government. This research was supported under Australian Research Council's Future Fellowship (Project No. FT120100760), Discovery Projects (Project No. DP140101337), and Laureate Fellowship (Project No. FL110100098) funding schemes.
[1] W. Reisdorf, F. Hessberger, K. Hildenbrand, S. Hofmann, G. Münzenberg, K.-H. Schmidt, J. Schneider, W. Schneider, K. Sümmerer, G. Wirth, J. Kratz, and K. Schlitt, Nucl. Phys. A 438, 212 (1985).

[2] J. R. Leigh, M. Dasgupta, D. J. Hinde, J. C. Mein, C. R. Morton, R. C. Lemmon, J. P. Lestone, J. O. Newton, H. Timmers, J. X. Wei, and N. Rowley, Phys. Rev. C 52, 3151 (1995).

[3] R. G. Stokstad, Z. E. Switkowski, R. A. Dayras, and R. M. Wieland, Phys. Rev. Lett. 37, 888 (1976).

[4] M. Beckerman, M. Salomaa, A. Sperduto, H. Enge, J. Ball, A. DiRienzo, S. Gazes, Y. Chen, J. D. Molitoris, and M. Nai-feng, Phys. Rev. Lett. 45, 1472 (1980).

[5] H. Esbensen, Nucl. Phys. A 352, 147 (1981).

[6] C. Dasso, S. Landowne, and A. Winther, Nucl. Phys. A 405 , 381 (1983).

[7] N. Rowley, G. Satchler, and P. Stelson, Phys. Lett. B 254, 25 (1991).

[8] M. Dasgupta, D. J. Hinde, N. Rowley, and A. M. Stefanini, Ann. Rev. Nucl. Part. Sci. 48, 401 (1998).

[9] B. B. Back, H. Esbensen, C. L. Jiang, and K. E. Rehm, Rev. Mod. Phys. 86, 317 (2014).

[10] A. B. Balantekin and N. Takigawa, Rev. Mod. Phys. 70, 77 (1998).

[11] K. Hagino and N. Takigawa, Prog. Theor. Phys. 128, 1061 (2012).

[12] C. Y. Wong, Phys. Rev. Lett. 31, 766 (1973).

[13] R. G. Stokstad, Y. Eisen, S. Kaplanis, D. Pelte, U. Smilansky, and I. Tserruya, Phys. Rev. Lett. 41, 465 (1978).

[14] C. R. Morton, M. Dasgupta, D. J. Hinde, J. R. Leigh, R. C. Lemmon, J. P. Lestone, J. C. Mein, J. O. Newton, H. Timmers, N. Rowley, and A. T. Kruppa, Phys. Rev. Lett. 72, 4074 (1994).

[15] A. M. Stefanini, D. Ackermann, L. Corradi, D. R. Napoli, C. Petrache, P. Spolaore, P. Bednarczyk, H. Q. Zhang, S. Beghini, G. Montagnoli, L. Mueller, F. Scarlassara, G. F. Segato, F. Soramel, and N. Rowley, Phys. Rev. Lett. 74, 864 (1995).
[16] A. Stefanini, G. Fortuna, R. Pengo, W. Meczynski, G. Montagnoli, L. Corradi, A. Tivelli, S. Beghini, C. Signorini, S. Lunardi, M. Morando, and F. Soramel, Nucl. Phys. A 456, 509 (1986).

[17] H. Timmers, L. Corradi, A. Stefanini, D. Ackermann, J. He, S. Beghini, G. Montagnoli, F. Scarlassara, G. Segato, and N. Rowley, Phys. Lett. B 399, 35 (1997).

[18] D. Bourgin, S. Courtin, F. Haas, A. M. Stefanini, G. Montagnoli, A. Goasduff, D. Montanari, L. Corradi, E. Fioretto, J. Huiming, F. Scarlassara, N. Rowley, S. Szilner, and T. Mijatović, Phys. Rev. C 90, 044610 (2014).

[19] C. L. Jiang, K. E. Rehm, B. B. Back, H. Esbensen, R. V. F. Janssens, A. M. Stefanini, and G. Montagnoli, Phys. Rev. C 89, 051603 (2014).

[20] C. L. Jiang, A. M. Stefanini, H. Esbensen, K. E. Rehm, S. Almaraz-Calderon, M. L. Avila, B. B. Back, D. Bourgin, L. Corradi, S. Courtin, E. Fioretto, F. Galtarossa, A. Goasduff, F. Haas, M. M. Mazzocco, D. Montanari, G. Montagnoli, T. Mijatovic, R. Sagaidak, D. Santiago-Gonzalez, F. Scarlassara, E. E. Strano, and S. Szilner, Phys. Rev. C 91, 044602 (2015).

[21] M. Dasgupta, D. J. Hinde, R. D. Butt, R. M. Anjos, A. C. Berriman, N. Carlin, P. R. S. Gomes, C. R. Morton, J. O. Newton, A. Szanto de Toledo, and K. Hagino, Phys. Rev. Lett. 82, 1395 (1999).

[22] L. Canto, P. Gomes, R. Donangelo, and M. Hussein, Phys. Rep. 424, 1 (2006).

[23] N. Keeley, R. Raabe, N. Alamanos, and J. Sida, Prog. Part. Nucl. Phys. 59, 579 (2007).

[24] N. Takigawa and H. Sagawa, Phys. Lett. B 265, 23 (1991).

[25] M. S. Hussein, Phys. Rev. C 44, 446 (1991).

[26] C. Dasso and R. Donangelo, Phys. Lett. B 276, 1 (1992).

[27] K. E. Zyromski, W. Loveland, G. A. Souliotis, D. J. Morrissey, C. F. Powell, O. Batenkov, K. Aleklett, R. Yanez, I. Forsberg, M. Sanchez-Vega, J. R. Dunn, and B. G. Glagola, Phys. Rev. C 55, R562 (1997). 
[28] P. A. DeYoung, B. Hughey, P. L. Jolivette, G. F. Peaslee, J. J. Kolata, V. Guimarães, D. Peterson, P. Santi, H. C. Griffin, J. A. Zimmerman, and J. D. Hinnefeld, Phys. Rev. C 58, 3442 (1998).

[29] M. Trotta, J. L. Sida, N. Alamanos, A. Andreyev, F. Auger, D. L. Balabanski, C. Borcea, N. Coulier, A. Drouart, D. J. C. Durand, G. Georgiev, A. Gillibert, J. D. Hinnefeld, M. Huyse, C. Jouanne, V. Lapoux, A. Lépine, A. Lumbroso, F. Marie, A. Musumarra, G. Neyens, S. Ottini, R. Raabe, S. Ternier, P. Van Duppen, K. Vyvey, C. Volant, and R. Wolski, Phys. Rev. Lett. 84, 2342 (2000).

[30] R. Raabe, J. L. Sida, J. L. Charvet, N. Alamanos, C. Angulo, J. M. Casandjian, S. Courtin, A. Drouart, D. J. C. Durand, P. Figuera, A. Gillibert, S. Heinrich, C. Jouanne, V. Lapoux, A. Lepine-Szily, A. Musumarra, L. Nalpas, D. Pierroutsakou, M. Romoli, K. Rusek, and M. Trotta, Nature (London) 431, 823 (2004).

[31] A. Lemasson, A. Navin, M. Rejmund, N. Keeley, V. Zelevinsky, S. Bhattacharyya, A. Shrivastava, D. Bazin, D. Beaumel, Y. Blumenfeld, A. Chatterjee, D. Gupta, G. de France, B. Jacquot, M. Labiche, R. Lemmon, V. Nanal, J. Nyberg, R. Pillay, R. Raabe, K. Ramachandran, J. Scarpaci, C. Schmitt, C. Simenel, I. Stefan, and C. Timis, Phys. Lett. B 697, 454 (2011).

[32] Z. Kohley, J. F. Liang, D. Shapira, R. L. Varner, C. J. Gross, J. M. Allmond, A. L. Caraley, E. A. Coello, F. Favela, K. Lagergren, and P. E. Mueller, Phys. Rev. Lett. 107, 202701 (2011).

[33] Z. Kohley, J. F. Liang, D. Shapira, C. J. Gross, R. L. Varner, J. M. Allmond, J. J. Kolata, P. E. Mueller, and A. Roberts, Phys. Rev. C 87, 064612 (2013).

[34] L. C. Vaz, J. M. Alexander, and G. R. Satchler, Phys. Rep. 69, 373 (1981).

[35] J. O. Newton, R. D. Butt, M. Dasgupta, D. J. Hinde, I. I. Gontchar, C. R. Morton, and K. Hagino, Phys. Rev. C 70 , 024605 (2004).

[36] C. E. Aguiar, V. C. Barbosa, C. H. Dasso, and R. Donangelo, Phys. Rev. C 46, R45 (1992).

[37] D. Vautherin and D. M. Brink, Phys. Rev. C 5, 626 (1972).

[38] D. Vautherin, Phys. Rev. C 7, 296 (1973).

[39] V. Rotival, K. Bennaceur, and T. Duguet, Phys. Rev. C 79, 054309 (2009).

[40] J. W. Negele, Rev. Mod. Phys. 54, 913 (1982).

[41] C. Simenel, Eur. Phys. J. A 48, 152 (2012).

[42] T. Skyrme, Philos. Mag. 1, 1043 (1956).

[43] V. E. Oberacker and A. S. Umar, Phys. Rev. C 87, 034611 (2013).

[44] K. A. Brueckner, J. R. Buchler, and M. M. Kelly, Phys. Rev. 173, 944 (1968).

[45] V. Y. Denisov and W. Nörenberg, Eur. Phys. J. A 15, 375 (2002).

[46] C. Simenel, M. Dasgupta, D. J. Hinde, and E. Williams, Phys. Rev. C 88, 064604 (2013).

[47] K.-H. Kim, T. Otsuka, and P. Bonche, J. Phys. G 23, 1267 (1997).

[48] M. Kortelainen, J. McDonnell, W. Nazarewicz, P.-G. Reinhard, J. Sarich, N. Schunck, M. V. Stoitsov, and S. M. Wild, Phys. Rev. C 85, 024304 (2012).

[49] P. Bonche, H. Flocard, and P. H. Heenen, Comput. Phys. Commun. 171, 49 (2005).

[50] K. Bennaceur, P. Bonche, and J. Meyer, C. R. Phys. 4, 555 (2003).
[51] M. Bender, K. Rutz, P.-G. Reinhard, J. A. Maruhn, and W. Greiner, Phys. Rev. C 60, 034304 (1999).

[52] S. Krieger, P. Bonche, H. Flocard, P. Quentin, and M. Weiss, Nucl. Phys. A 517, 275 (1990).

[53] G. Scamps and D. Lacroix, EPJ Web Conf. 86, 00042 (2015).

[54] R. A. Broglia and A. Winther, in Heavy Ion Reaction Lecture Notes, Vol. I: Elastic and Inelastic Reactions (Benjamin/ Cummings, Reading, MA, 1981), p. 114.

[55] C. H. Dasso and G. Pollarolo, Phys. Rev. C 68, 054604 (2003).

[56] Ş. Mişicu and H. Esbensen, Phys. Rev. Lett. 96, 112701 (2006).

[57] C. L. Jiang, H. Esbensen, K. E. Rehm, B. B. Back, R. V. F. Janssens, J. A. Caggiano, P. Collon, J. Greene, A. M. Heinz, D. J. Henderson, I. Nishinaka, T. O. Pennington, and D. Seweryniak, Phys. Rev. Lett. 89, 052701 (2002).

[58] M. Dasgupta, D. J. Hinde, A. Diaz-Torres, B. Bouriquet, C. I. Low, G. J. Milburn, and J. O. Newton, Phys. Rev. Lett. 99, 192701 (2007).

[59] H. D. Wohlfahrt, E. B. Shera, M. V. Hoehn, Y. Yamazaki, and R. M. Steffen, Phys. Rev. C 23, 533 (1981).

[60] L. Vermeeren, R. E. Silverans, P. Lievens, A. Klein, R. Neugart, C. Schulz, and F. Buchinger, Phys. Rev. Lett. 68, 1679 (1992).

[61] E. Caurier, K. Langanke, G. Martínez-Pinedo, F. Nowacki, and P. Vogel, Phys. Lett. B 522, 240 (2001).

[62] J. Błocki and H. Flocard, Phys. Lett. B 85, 163 (1979).

[63] J. W. Negele, S. E. Koonin, P. Möller, J. R. Nix, and A. J. Sierk, Phys. Rev. C 17, 1098 (1978).

[64] P. Bonche, S. Koonin, and J. W. Negele, Phys. Rev. C 13, 1226 (1976).

[65] J. A. Maruhn, P. G. Reinhard, P. D. Stevenson, J. R. Stone, and M. R. Strayer, Phys. Rev. C 71, 064328 (2005).

[66] A. S. Umar and V. E. Oberacker, Phys. Rev. C 71, 034314 (2005)

[67] T. Nakatsukasa and K. Yabana, Phys. Rev. C 71, 024301 (2005).

[68] J. Maruhn, P.-G. Reinhard, P. Stevenson, and A. Umar, Comput. Phys. Commun. 185, 2195 (2014).

[69] A. S. Umar, M. R. Strayer, and P. G. Reinhard, Phys. Rev. Lett. 56, 2793 (1986).

[70] C. Simenel and B. Avez, Int. J. Mod. Phys. E 17, 31 (2008).

[71] K. Washiyama and D. Lacroix, Phys. Rev. C 78, 024610 (2008).

[72] C. Simenel, R. Keser, A. S. Umar, and V. E. Oberacker, Phys. Rev. C 88, 024617 (2013).

[73] A. S. Umar, C. Simenel, and V. E. Oberacker, Phys. Rev. C 89, 034611 (2014).

[74] G. Scamps and D. Lacroix, Phys. Rev. C 88, 044310 (2013).

[75] G. Scamps, C. Simenel, and D. Lacroix, Phys. Rev. C 92, 011602 (2015).

[76] A. Bulgac, P. Magierski, K. J. Roche, and I. Stetcu, Phys. Rev. Lett. 116, 122504 (2016).

[77] A. Wakhle, C. Simenel, D. J. Hinde, M. Dasgupta, M. Evers, D. H. Luong, R. du Rietz, and E. Williams, Phys. Rev. Lett. 113, 182502 (2014).

[78] A. S. Umar, V. E. Oberacker, and C. Simenel, Phys. Rev. C 92 , 024621 (2015).

[79] S. Fracasso, E. B. Suckling, and P. D. Stevenson, Phys. Rev. C 86, 044303 (2012).

[80] G. Scamps and D. Lacroix, Phys. Rev. C 89, 034314 (2014).

[81] P. G. Reinhard, L. Guo, and J. A. Maruhn, Eur. Phys. J. A 32, 19 (2007).

[82] C. Simenel and P. Chomaz, Phys. Rev. C 80, 064309 (2009).

[83] P. Chomaz, N. V. Giai, and S. Stringari, Phys. Lett. B 189, 375 (1987). 
[84] B. Avez and C. Simenel, Eur. Phys. J. A 49, 76 (2013).

[85] T. Kibédi and R. H. Spear, At. Data Nucl. Data Tab. 80, 35 (2002).

[86] S. Raman, C. Malarkey, W. Milner, C. Nestor Jr., and P. Stelson, At. Data Nucl. Data Tab. 36, 1 (1987).

[87] K. Hagino, N. Rowley, and A. Kruppa, Comput. Phys. Commun. 123, 143 (1999).

[88] K. Hagino, N. Takigawa, M. Dasgupta, D. J. Hinde, and J. R. Leigh, Phys. Rev. Lett. 79, 2014 (1997).

[89] M. Evers, M. Dasgupta, D. J. Hinde, D. H. Luong, R. Rafiei, R. du Rietz, and C. Simenel, Phys. Rev. C 84, 054614 (2011).

[90] A. Trzcińska, E. Piasecki, A. Amar, W. Czarnacki, N. Keeley, M. Kisieliński, S. Kliczewski, M. Kowalczyk, B. Lommel, M. Mutterer, R. Siudak, A. Stolarz, I. Strojek, G. Tiourin, and W. H. Trzaska, Phys. Rev. C 93, 054604 (2016).

[91] I. Thompson, M. Nagarajan, J. Lilley, and B. Fulton, Phys. Lett. B 157, 250 (1985)

[92] G. Pollarolo, Acta Phys. Pol. B 44, 407 (2013).

[93] A. V. Karpov, V. A. Rachkov, and V. V. Samarin, Phys. Rev. C 92, 064603 (2015).

[94] G. Scamps and K. Hagino, Phys. Rev. C 92, 054614 (2015).

[95] S. E. Koonin, K. T. R. Davies, V. Maruhn-Rezwani, H. Feldmeier, S. J. Krieger, and J. W. Negele, Phys. Rev. C 15, 1359 (1977).

[96] C. Simenel, Phys. Rev. Lett. 105, 192701 (2010).

[97] S. Ayik, O. Yilmaz, B. Yilmaz, A. S. Umar, A. Gokalp, G. Turan, and D. Lacroix, Phys. Rev. C 91, 054601 (2015).
[98] G. Scamps and D. Lacroix, Phys. Rev. C 87, 014605 (2013).

[99] K. Sekizawa and K. Yabana, Phys. Rev. C 88, 014614 (2013)

[100] G. Scamps, V. V. Sargsyan, G. G. Adamian, N. V. Antonenko, and D. Lacroix, Phys. Rev. C 91, 024601 (2015).

[101] Sonika, B. J. Roy, A. Parmar, U. K. Pal, H. Kumawat, V. Jha, S. K. Pandit, V. V. Parkar, K. Ramachandran, K. Mahata, A. Pal, S. Santra, A. K. Mohanty, and K. Sekizawa, Phys. Rev. C 92, 024603 (2015).

[102] D. Bourgin, C. Simenel, S. Courtin, and F. Haas, Phys. Rev. C 93, 034604 (2016).

[103] C. Simenel and A. S. Umar, Phys. Rev. C 89, 031601 (2014).

[104] C. Simenel, D. J. Hinde, R. du Rietz, M. Dasgupta, M. Evers, C. J. Lin, D. H. Luong, and A. Wakhle, Phys. Lett. B 710, 607 (2012).

[105] V. E. Oberacker, A. S. Umar, and C. Simenel, Phys. Rev. C 90, 054605 (2014).

[106] D. J. Kedziora and C. Simenel, Phys. Rev. C 81, 044613 (2010).

[107] K. Washiyama, D. Lacroix, and S. Ayik, Phys. Rev. C 79, 024609 (2009).

[108] A. S. Umar, V. E. Oberacker, J. A. Maruhn, and P. G. Reinhard, Phys. Rev. C 80, 041601 (2009).

[109] K. Sekizawa and K. Yabana, Phys. Rev. C 90, 064614 (2014).

[110] Y. Hashimoto and G. Scamps, Phys. Rev. C 94, 014610 (2016).

[111] J. R. Stone, P. A. M. Guichon, P. G. Reinhard, and A. W. Thomas, Phys. Rev. Lett. 116, 092501 (2016). 